\title{
Neural Correlates of Causal Inferences and Semantic Priming in People with Williams Syndrome: An fMRI Study
}

\author{
Ching-Fen $\mathrm{Hsu}^{1,2, *}$ \\ ${ }^{1}$ School of Foreign Languages; ${ }^{2}$ Research Center for Language Pathology and Developmental Neuroscience, \\ Hunan University, Lushan Road (S), Yuelu District, Changsha, Hunan Province, 410082, China
}

\begin{abstract}
This study aimed at examining the ability of causal inferences and semantic priming of people with Williams syndrome (WS). Previous studies pointed out that people with WS showed deviant sentence comprehension, given advantageous lexical semantics. This study investigated the impairment in connecting words in the semantic network by using neuroimaging techniques to reveal neurological deficits in the contextual integration of people with Williams syndrome. Four types of word pairs were presented: causal, categorical, associative, and functional. Behavioural results revealed that causal word pairs required heavier cognitive processing than functional word pairs. Distinct neural correlates of semantic priming confirmed atypical semantic linkage and possible cause of impairment of contextual integration in people with WS. The findings of normal behaviours and atypical neural correlates in people with WS provide evidence of atypical development resulted from early gene mutations.
\end{abstract}

Keywords: Neuroimaging, Williams syndrome, causal inference, semantic priming, atypical development.

\section{INTRODUCTION}

Williams syndrome (WS) is a rare disorder with genetic deficits on chromosome $7 \mathrm{q} 11.23$ in the aetiology of 1 in 7500 live births [1, 2]. Due to gene deficits, people with WS are mentally retarded with an average IQ of 55 and visuospatial impairments [3]. But people with WS are relatively good at linguistic abilities, including lexical-semantic knowledge and conditional sentences [3-6]. People with WS are unique in the cognitive processing style of focusing on local elements but ignoring global configuration similar to weak central coherence in people with autism spectrum disorders [7]. This cognitive style has been observed not only in the verbal domain but also in the nonverbal domain. In the verbal domain, people with WS are relatively good at lexical semantics but impaired in contextual integration. People with WS are deficient at integrating words into sentences. This deficiency may be due to an impaired ability to connect words. This current study aimed at examining differences in processing distinct types of semantic relatedness at neurological level in people with WS. It was hypothesised that distinct neural bases would be identified in people with WS from healthy controls while responding to different types of words. This study further clarified linguistic classifications regarding semantic relatedness rather than a vague mix-up of terminology in priming tasks.

Contextual coherence is the competence to integrate partial information as a meaningful gist. A

*Address correspondence to this author at the Research Center for Language Pathology and Developmental Neuroscience, Hunan University, Lushan Road (S), Yuelu District, Changsha, Hunan Province, 410082, China;

Tel: +86-0731-88821135; E-mail: chinghsu@hnu.edu.cn series of studies on contextual coherence in people with WS have been conducted [8-15]. Different linguistic units were examined in these studies, including words, propositions, and sentences. Investigating contextual coherence in people with WS originated from the characteristic of focusing on local elements but ignoring global configurations. People with WS have difficulty integrating parts into whole or gist in information processing, similar to people with autism spectrum disorders. This difficulty is termed as weak central coherence in cognitive processing style in verbal and nonverbal domains [3, 16-19].

Hsu et al. [20] investigated word-level integration in people with WS by using the false memory paradigm [21]. Word lists with semantically related associates were presented aurally first, and participants were asked to make judgments whether a word was presented before. All the associates were Chinese disyllabic words and related to each other within a word list under a theme. For instance, scarf, hot pot, sweater, glove, warm, coat, hot soup, snow, and many others in the word list pointing to the winter theme. All the associates were generated by college students. It was predicted that the unpresented themes of word lists would be falsely recognised as the presented words because of the automatic integration of contextual information. The results revealed high falsepositive rates to the themes in chronological age (CA)matched controls. People with WS showed misrecognition rates as their mental age (MA)-matched controls, suggesting a developmental delay in processing word-level associated words in contextual integration. In Hsu et al.'s study [20], behavioural responses were measured, and their brainwaves were 
recorded simultaneously. The neurological results yielded interesting patterns in CA controls and WS individuals. While the CA controls processed themes as presented old words that were different from new words, the individuals with WS processed themes as new words that were distinct from old words. These results implied that people with WS processed semantically integrated concepts as semantically unrelated words, whereas healthy controls processed the same semantically integrated concepts as the presented semantically related associates, indicating automatic integration of semantically related words in the semantic network. However, response latency and accuracy rates of people with WS and healthy controls were in a similar normal range. This finding is termed as asymmetry of brain and behaviour, referring to normal-like behavioural performances but atypical neurological processing. This finding is an extremely important breakthrough in the research of developmental disabilities, implying possible interventions to improve cognition and language abilities in people with developmental disabilities. Pinheiro et al. [22] confirmed atypical neurological processing in people with WS in comprehending anomalous sentences. Hsu and colleagues [20] uncovered that people with WS performed like healthy controls in word-level integration behaviourally but not neurologically. Together, asymmetry of brain and behaviour has been confirmed in people with WS.

To further examine the difficulty of contextual integration in people with WS, Hsu [23] investigated their ability in linking different types of semantic priming: synonyms (clap hands, 拍手[pai1-shou3] vs. clap hands, 鼓掌[gu3-zhang3]), categorically related words (lipstick, 口紅[kong3-hong2] vs. facial powder, 粉餅[fen3-bing3]), and functionally related words (cleaning cloth, 抹布[mo3-bu4] vs. water basket, 水桶[shui3-tong3]). Three experiments were conducted with each of the three types of semantic priming taking associative words (spring, 春天[chun1-tian1] vs. flowerwatching, 賞花[shang3-hua1]) and unrelated words (coat, 外套[wai4-tao4] vs. cable, 電線[dian4-xian4]) as control words. Participants were instructed to listen to each word pair and decide whether the pair were semantically related. The results revealed that participants with WS erred more with functional words than CA controls, suggesting people with WS had difficulty processing functional words. No difference between groups in processing synonyms, categorical, and associative words was observed. Participants with WS correctly rejected unrelated word pairs as semantically unrelated words. While the CA group was faster in reaction times to categorical words than associative words, individuals with WS failed to show this difference. The response latencies to the categorical words of CA controls were as quick as the ones to the unrelated words, whereas WS individuals processed slower to categorical words than the unrelated words. Moreover, people with WS showed normal-like performances in comprehending synonyms and functionally related words as in finding by Tyler et al. [5]. Together, categorically related words were processed distinctly in people with WS from healthy controls.

In addition to impairment of word-level integration in people with WS, the proposition-level deficit was demonstrated in this clinical population [9]. In the study by Hsu and Tzeng [9], sentences embedded with propositions in various numbers were presented to the participants in a learning phase; later, in a recognition phase, the participants were asked to make recognition judgments whether they had heard the sentences before [24-26]. Four parent sentences were decomposed based on embedded propositions and presented, e.g., $A$ wild wolf in the forest caught a rabbit which was eating carrots in the brushwood. Then, four propositions were composed of the parent sentence as follows: a wild wolf was in the forest, a rabbit was in the brushwood, a wild wolf caught a rabbit, and a rabbit was eating carrots. The parent sentences were presented in the recognition phase only. It was predicted that the recognition rates would be a function of the embedded propositions in sentences. The sentences with more propositions embedded had a higher possibility of being misrecognised. The CAmatched typically developing controls and the MAmatched typically developing controls showed the same pattern as the predictions. The CA controls showed false positives to four-proposition sentences; the MA controls showed false positives to sentences larger than one proposition. The individuals with WS failed to show the patterns as the healthy controls and had difficulty in differentiating old sentences from new ones. These findings suggested that healthy controls integrated propositions automatically, whereas people with WS faced difficulty in doing so.

Pinheiro et al. [22] reported atypical processing of sentences in people with WS. Sentences were aurally presented along with congruent or incongruent words in the final position. The participants were asked to make a judgment whether the word made good completion of each sentence by responding yes or no. 
While behavioural responses of reaction times and accuracy were measured, brainwave responses were recorded by event-related potentials. The healthy controls showed higher accuracy and congruency effect compared to people with WS. As with brainwave responses, healthy controls showed more negative amplitudes to incongruent sentences on N100, while individuals with WS failed to show this pattern. Instead, people with WS showed a more positive amplitude on P200 in the central region of the right hemisphere $(\mathrm{RH})$ compared to the left hemisphere (LH). The presence of small N100 and larger P200 in people with WS in the verbal study was compatible with a nonverbal study on the face processing of people with WS [27]. No difference in $\mathrm{N} 400$ between groups was observed. However, a difference emerged in 500-600 ms, suggesting that people with WS showed more positive amplitudes in the frontal and central regions than healthy controls in responding to mismatched sentences.

Further analyses of $600-700 \mathrm{~ms}$ revealed that people with WS processed more positively in the right hemisphere than in the left hemisphere. The index of P600 denoting late integration of lexical items into sentences differentiated the healthy controls from people with WS in integrating words into sentences. Hence, it is proposed that people with WS have difficulty in integrating or connecting semantically related words. Neville et al. [28] reported atypical integration of associative words into sentences in people with WS; for example, She took a cup of coffee with sugar and _ [cream versus socks]. The incongruent words were compared with semantically congruent words in sentences. The results revealed compromised $\mathrm{N} 400$ brain responses to the incongruent sentences, suggesting people with WS processed semantically related words differently. Therefore, based on the studies by Neville et al. [28] and Pinheiro et al. [22], it can be said that in people with WS, the difficulty in sentence comprehension might be mainly due to the deficiency in linking of semantic words in sentences.

To figure out the cause of integration difficulty in people with WS, a study on causal reasoning in context was examined [10]. People with WS were aurally presented scenarios embedded with ambiguous words in sentence-final positions and had to respond to a comprehension question at the end of the scenario. Only through the correct interpretation of each ambiguous word, the comprehension question could be responded to correctly. Each ambiguous word could be interpreted in its figurative and literal meaning. For instance, po1 leng3 shuei3 (潑冷水, translated character by character as 'pour cold water') has the figurative meaning as "to dampen one's enthusiasm" and the literal meaning as "to pour cold water on someone". The participants were asked to listen to a scenario served as the consequence of the story, for example, "Sponge Bob would like to eat the candies on a shelf. He asked for help from Squidward Tentacles. But Squidward Tentacles dampened Sponge Bob's enthusiasm". The cause scenario was then presented to the participants: "Sponge Bob is not tall enough to reach the candies on the shelf'. A comprehension question was followed after the entire story: "What did Squidward Tentacles do?". Three alternatives were presented to the participants (1) (figurative meaning) Squidward Tentacles did not help Sponge Bob to get the candies, (2) (literal meaning) Squidward Tentacles poured cold water on Sponge Bob, (3) (unrelated meaning) Sponge Bob likes to take a bath with cold water. The participants had to choose one out of the three options given to them indicated continuity and completeness in communicating the information. This task is termed as forwarding inference because causal inferences are made from causes to consequences.

Another type of causal inferences, from consequences to causes, termed as backward inference, was tested on people with WS. The procedure was similar to forwarding inference, but the presented sequence was reversed. For example, the figurative meaning of er3 bian1 feng1 (耳邊風) is "being inattentive to a suggestion or command," and the literal meaning is "a wind blowing past the ears". The participants were presented a cause scenario: "Daxung failed the exam this time. His mother often reminded him to study hard, but Daxung was inattentive to his mother's reminders" and a consequence scenario: "Daxung regretted not paying attention to his mother's reminders". A comprehension question, "What did Daxung do?" was then asked. The participants were required to pick one as the correct interpretation, based on the context, out of the three choices presented (1) (figurative meaning) "Daxung did not take his mother's words seriously", (2) (literal meaning) "There was a wind blowing by Daxung's ears", (3) (unrelated meaning) "Daxung's ears were itchy because of the blowing wind". The correct answer could be chosen only when the intended meaning was reached. The results revealed that people with WS performed similarly as healthy MA-matched controls choosing more figurative meanings, over-literal meanings or unrelated meanings. However, individuals with WS 
selected more unrelated meanings than MA-matched controls, suggesting deviant comprehension of ambiguous words or more easily being influenced by superficial expressions. The patterns of performances were similar in forward and backward inferences, but backward inferences were easier than forward inferences for the MA controls and WS individuals. The findings revealed that causal inference takes time to develop from childhood to adulthood. Another study on people with Down Syndrome (DS) by Hsu [29], using the same stimuli and procedures, revealed that people with DS were deviant in causal inferences compared to healthy controls. The results indicated that distinct genotypes contributed to different phenotypes in people with neurodevelopmental disorders [30].

Causal inference, together with semantic priming, is a fundamental cognitive ability to reach contextual coherence. This fMRI study aimed at finding out the neural correlates of distinct types of semantic priming in people with WS. Further, the aim was to find supporting evidence to the asymmetry of brain and behaviour in people with WS as in other developmental disabilities. Through comparisons with typically developing controls, possible causes of weak contextual coherence could be identified in WS individuals. It was hypothesised that people with WS would go through difficulty in processing functionally related words compared to other types of word pairs, as the previous study revealed. Supporting evidence to the asymmetry of brain and behaviour in responding to other types of semantically related words would be observed in people with WS, suggesting normal behaviours with atypical neural correlates in people with WS. Distinct neural correlates of people with WS and healthy controls in causal inferences and semantic priming at the lexical level would emerge.

\section{METHODS}

\section{Participants}

Seven individuals with WS were recruited. They were diagnosed in hospitals with genetic deficits on chromosome $7 q 11.23$, with missing genes averagely from 15 to 22 at various ages. The individuals with WS were matched individually by gender and $C A$ to typically developing controls. The average CA of these two groups did not show any significant differences $[t(6)=0.578, p=0.584]$; hence, any differences at behavioural or neurological level could not be accounted for by their CA. This is the ideal matching method of study for examining brain activations for different types of words in semantic relatedness in people with WS and healthy controls while considering the compatible brain structures. The MA of the participants with WS differed from the CA of healthy controls and the participants with WS [MA in people with WS vs. CA in healthy controls, $t(6)=6.11, p=$ 0.001 , MA in people with WS vs. CA in people with WS, $t(6)=11.001, p<0.001]$. Moreover, since participants with WS were matched individually in gender and age with healthy controls, the differences at behavioural and neurological levels could not have been due to the intellectual functioning between the groups. There was no need to recruit another group matched in MA as the traditional matching method because brains were still in the developing stage in this group. It made no sense to compare the developing brains of the MA group with those of the individuals with WS, who were already in their CA-level development. Still, the MA of the participants with WS were obtained by using the Wechsler Scale of Intelligence for Children (WSIC-IV, Chen \& Chen [31]) for those younger than 16 years, and the Wechsler Adult Intelligence Scale (WAIS-III, Chen \& Chen [32]) for those older than 16 years. Since parents or guardians of WS participants were concerned about the safety of fMRI scanning, explanation in oral and written forms were given prior to the experiment. Each participant with WS had a health check-up with a doctor familiar with the patient's development before scanning. Consent forms were signed by the participants with WS and their parents or guardians before the experiments began. The typically developing controls under 20 years old and their guardians signed the consent forms. Before entering the scanning room, the screening criteria met the rigorous regulations that all participants should not carry any metal objects, metal materials, and a cardiac regulator in/on their bodies. The study was approved by the Institutional Review Board of National Chengchi University in Taiwan (NCCU-REC-201609-I044). The background information of the participants is listed in Table 1.

\section{Pre-Experimental Procedure before Imaging Task}

Two files, one audio file of fMRI scanning noise and the other of the experiment procedure, were sent to the WS participants and their invited parents before their visit to the centre. The procedure demonstrated the sequence of must-do steps before the final scanning: (1) lie on the scanner bed, (2) earplugs in to prevent the scanner noise, (3) head covered with a helmet to ensure no movement, and (4) practice button-pressing based on the experimental instructions. All participants 
Table 1: Background Information of Participants in the WS Group and the CA Control Group

\begin{tabular}{|c|c|c|c|c|c|c|}
\hline Group & N & F:M & Mean CA (s.d.) & Range & Mean MA (s.d.) & Range \\
\hline \hline WS & 7 & $3: 4$ & $16 ; 20(1 ; 89)$ & $12 ; 01-17 ; 11$ & $7 ; 31(2 ; 04)$ & $4 ; 08-11 ; 00$ \\
\hline CA & 7 & $3: 4$ & $18 ; 08(3 ; 01)$ & $15 ; 04-24 ; 10$ & & \\
\hline
\end{tabular}

stayed inside the scanner safely with a microphone connected for talking to the experimenters outside the scanning room. On the testing day, the participants with WS received a mock practice with no magnet before the actual experiments began with a $3 T$ scanner.

\section{Word-Generating Task}

To examine neural correlates of causal inferences and semantic priming, four types of word pairs were included in this study: causal, associative, categorical, and functional. All were disyllabic Chinese words. The causal word pairs indicated words binding in the relationship of cause and effect. For example, earthquake (地震[di4-zhen4]) vs. out of electricity (停電[ting2-dian4]). The order in causal pairs could not be switched because a causal relationship exists. The first word is the cause behind the second word. As the example shows, an earthquake could possibly be a cause for the consequence of losing electricity. But the reverse is not true. In associative word pairs, the role binding is not necessary, and the two words can be in any order, for example, doctor (醫生[yi1-sheng1]) vs. nurse (護士[hu4-shi4]). Categorical word pairs are objects in the same category, like a spoon (湯匙[tang1chi1]) vs. chi chops (筷子[kuai4-zi]), referring to catering tools. Functional word pairs are objects related to specific tools used in certain activities like goggles (蛙鏡[wa1-jing4]) vs. swimming (游泳[you2-yong3]). Unrelated words were included as fillers. Sixty word pairs were generated for each type and each pair was rated by 60 college students (mean age $=20.09$ years, $\mathrm{SD}=1.05$, range $=19.00-22.11,27 \mathrm{M} / 33 \mathrm{~F}$ ). Each rater made judgments in associative strength of word pairs from highest 5 to lowest 1 . The results showed a high average of semantic closeness: 4.46 for causal related pairs $(S D=0.22, S E=0.038), 4.40$ for associative related pairs $(S D=0.18, S E=0.032), 4.25$ for categorical related pairs $(S D=0.25, S E=0.044), 4.48$ for functional related pairs $(S D=4.48, S E=0.028)$, and 1.12 for low closeness for unrelated words (SD = 0.05 , SE $=0.010)$. The analyses of variances with repeated measures revealed a significant effect of word types $[F(4,124)=4486, p<0.001]$. All differences between groups were significant at $p<.001$ level (except the difference between causal word pairs and functional word pairs did not reach significance, $p=$ 0.23). The unrelated words were significantly lower than words with causal word pairs and associative word pairs. The selection of words for experimental stimuli was based on the criteria defined for college students aged 19 to 22 years.

\section{Materials and Design}

Four blocks with 40 trials each were presented for all participants. Each block contained 32 target word pairs and 8 unrelated filler pairs. Participants were asked to make a judgment to each word pair whether the two words were related causally, associatively, categorically, or functionally. Before scanning, all participants practised trials to familiarise themselves with the procedure, including instruction and counterbalanced button pressing. A block design was employed to maximise the experimental effect, and trials were randomly presented.

\section{Procedures}

A fixation was displayed on the screen centre for $1000 \mathrm{~ms}$. Both the prime and the target of a word pair appeared for $3 \mathrm{~s}$ with zero interstimulus interval. The values of jitter were from $2000 \mathrm{~ms}$ to $4000 \mathrm{~ms}$, and the average was $3000 \mathrm{~ms}$. In the imaging study, each scan took $2 \mathrm{~s}$. Each trial required 5 scans (10 s in total). The participants pressed buttons, with an index finger or middle finger in a counterbalanced method, to indicate yes or no. They had received practice trials before entering the scanner. The instruction was given as follows: "It's nice to have you here to play this game with us. You see, there is a computer in front of you, and two Chinese disyllabic words are going to be displayed on the screen one at a time. You should read and understand each of them from left to the right. The first word is going to be shown for a short while, and the second word will follow. Upon presenting the second word, you should press the button on the keyboard to show your understanding about the two words whether they are related in [association (for block 1), category (for block 2), cause and effect (for block 3), and function (for block 4), the name of the 
block given depending on true condition that the participant was taking]. If the two words are related in (the name of the block), press the green button; if the two words are not related, press the red button. Are you ready to play the game? Let us do some practice." Functional brain images (T2) were scanned first (27 mins), and structural brain images (T1) (7 mins) followed for each participant.

The procedures were exactly the same as the experimental file sent to the participants beforehand. Participants lay on the scanning bed with their heads fixated by a helmet. To decrease tremendous scanning noise, all participants wore earplugs before entering the room. They were informed to squeeze the ball in their non-dominant hands in case they felt uncomfortable and wanted to stop experiments or leave the scanning room. Before the actual experiments began, participants were told not to move during scanning in experiments. Critical head motions were recorded simultaneously, and the values were the indexes for the experimenter to adjust the participants' positions in the scanner at any time.

Before scanning, a mock practice for the individuals with WS was arranged. Each experiment lasted about an hour. Due to attention limit of the participants with WS, the blocks probing causal and associative word pairs were presented before categorical and functional blocks to some of them (3 out of 7). Healthy controls and other WS individuals were presented the fixed order of categorical, associative, causal, and functional blocks. This subtle difference in presentation order of the blocks between two groups was to maximise the experimental effect in causal inferences and semantic priming at a neurological level for people with WS.

\section{Neuroimaging Data Acquisition and Processing}

Imaging data was collected by using Siemens MAGNETOM Skyra 3 Tesela scanner. Functional brain images (T2) were scanned with the echo planar imaging (EPI) parameters as follows: time of repetition $(T R)=2000 \mathrm{~ms}$, time to encode $(T E)=30 \mathrm{~ms}$, flip angle $=90^{\circ}$, field of view $($ FOV $)=220 \times 220 \mathrm{~mm}$, acquisition matrix $=64 \times 64$, resolution $=3.4 \times 3.4 \times 4$ $\mathrm{mm}$, and slice number $=36$. Structural brain images (T1) were scanned in the same session with the following parameters: $\mathrm{TR}=3500 \mathrm{~ms}$, TE $=3.37 \mathrm{~ms}$, flip angle $=7^{\circ}, \mathrm{TI}=1100 \mathrm{~ms}, \mathrm{FOV}=256 \times 256 \mathrm{~mm}$, acquisition matrix $=256 \times 256$, resolution $=1 \times 1 \times 1$ $\mathrm{mm}$, and slice number $=192$.
Brain images were processed using Statistical Parametric Mapping (SPM 12, (Wellcome Department of Imaging Neuroscience, London, UK) in standard analysing procedures. The acquired data went through individual pre-processing, including motion correction, slice timing, realignment, co-registration, normalisation with the template, and smoothing. After finishing the $1^{\text {st }}$ level analyses, individual data were put together for the $2^{\text {nd }}$ level group analyses. Three steps were analysed: model specification, model estimation, and statistics. In the last step of statistical analyses, subtraction of the activated brain regions of the healthy controls from the WS participants in each type of semantic relatedness was applied. The results indicated that WS participants were inactive in causal inferences and semantic priming compared to the healthy controls.

\section{Behavioural Results}

A pre-scanning behavioural study measuring reaction times and accuracy was employed to investigate the priming effect of the typically developing controls. Twenty college students were recruited (mean age $=20.44$ years, $S D=1.12$, age range $=18.08-$ $23.04,5 \mathrm{M} / 15 \mathrm{~F})$. The behavioural study was completed in a quiet room of the imaging centre with a laptop. No individuals with WS were tested in the behavioural study due to the limited number of the rare disorder, to avoid possible practice effect later in scanning.

Only correct responses were put into analyses, and the average of responses in each type of semantic relatedness was calculated and compared. Accuracy of each word type taken as the within-participants factor and groups as the between-participants factors were subjected to the repeated measure analyses of variance. The results showed significant difference among groups $\left[F(3,57)=3.05, p=0.036, \eta^{2}=0.138\right]$, suggesting participants responded most correctly to functional word pairs $(0.977, S D=0.010)$ compared to causal word pairs $(0.933, \mathrm{SE}=0.023)$ at $p=0.024$ and categorical word pairs $(0.952, \mathrm{SE}=0.016)$ at $p=0.042$. No difference was observed between functional words and associative words $(0.947, \mathrm{SE}=0.021)$, and no difference emerged among other word pairs. In other words, causal word pairs were processed similar to categorical word pairs and associative word pairs, but differently from functional word pairs.

The analyses of reaction times reached significance $\left[F(3,57)=5.015, p=0.004, \eta^{2}=0.209\right]$. The causal word pairs $(1595 \mathrm{~ms}, \mathrm{SD}=286 \mathrm{~ms})$ were responded longer than categorical word pairs $(1375 \mathrm{~ms}, \mathrm{SD}=341$ 
$\mathrm{ms})$ at $p<0.001$ and functional word pairs $(1440 \mathrm{~ms}$, $\mathrm{SD}=322 \mathrm{~ms})$ at $p=0.012$. Causal word pairs were not different from associative word pairs $(1521 \mathrm{~ms}, \mathrm{SD}=$ $356 \mathrm{~ms})$. The categorical word pairs were responded faster than the associative word pairs at $p=0.004$. It is interesting to note that causal word pairs were responded less accurately and were processed slower than functional word pairs even with no difference in semantic rating, suggesting that these two types of words are truly distinct in semantic content and the healthy adults processed functionally related words with ease. Causal word pairs required more cognitive load in processing bond of cause and effect. Hence, causal word pairs were responded with longer reaction times than functional and categorical word pairs, and with a lower percentage of correct response compared to functional word pairs.

Behavioural data of the neuroimaging study of WS individuals and healthy controls who were newly recruited (see Table 1) were analysed with the repeated measure ANOVA, taking types of semantic relatedness as a within-participants factor and groups as a between-participants factor. The dependent variables of reaction times and accuracy yielded no interactions of the two factors. The main effect of word types in accuracy reached significance $[F(3,36)=$ 3.374, $\left.p=0.029, \eta^{2}=0.219\right]$, suggesting more accurate responses of causal word pairs $(0.844, \mathrm{SD}=$ $0.042)$ at $p=0.03$ and functional word pairs $(0.837$, SD $=0.037)$ at $p=0.005$ than associative word pairs (0.728, SD $=0.042)$. No difference was observed among other word types. The causal word pairs were processed similar to the categorical and functional word pairs. The CA controls $(0.93, S D=0.048)$ were more accurate than the individuals with WS $(0.68, S D=$ 0.048 ) [main effect of group, $F(1,12)=13.93, p=$ $\left.0.003, \eta^{2}=0.537\right]$. Binominal tests revealed that the percentages of accuracy in each word type in people with WS were against chance $(p=0.016)$.

Moreover, the CA controls responded to all types of words faster than individuals with WS $[F(1,12)=520, p$ $\left.<0.001, \eta^{2}=0.977\right]$. No differences in types of semantic priming in accuracy and in reaction times were observed. Figure 1 shows the percent of the accuracy of the two groups to the four types of semantic meanings. Figure 2 displays the response latency of the two groups to the same types of semantic meanings.

Together, these behavioural findings suggested that people with WS performed similarly as the CA controls in causal inferences and semantic priming. However, the neural correlates of causal inferences and semantic priming were different in people with WS.

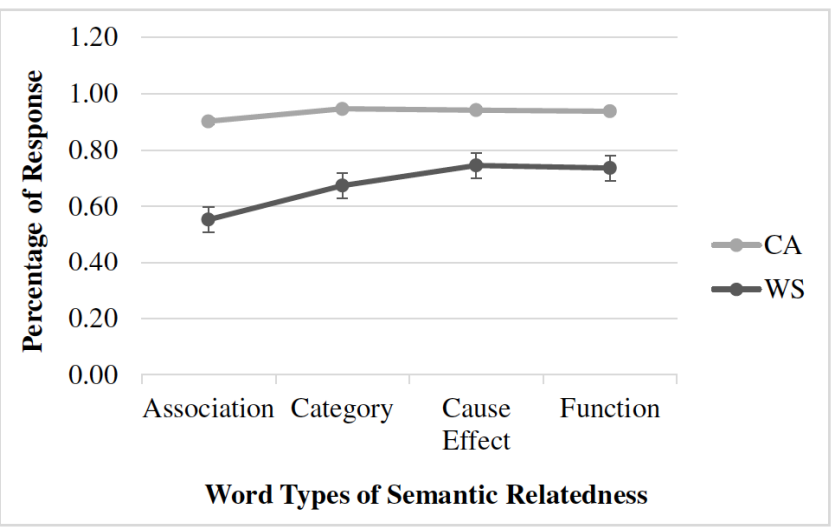

Figure 1: Percentage of the accuracy of the four types of words in the CA-matched controls and people with Williams Syndrome.

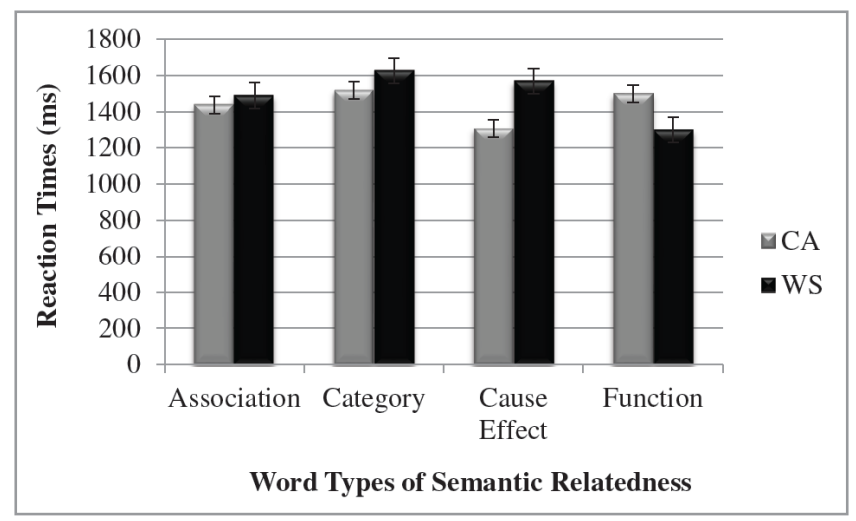

Figure 2: Response latencies of the four types of words in the CA-matched controls and people with WS.

\section{Neuroimaging Results}

This study aimed at figuring out the neural correlates of causal inferences and semantic priming in people with WS. The causal word pairs activated the left superior frontal gyrus and right frontal sub-gyral precentral areas (Figure 3). The categorical word pairs activated the right parietal precuneus (Figure 4). The functional word pairs activated the right insula (Figure 5). The associative word pairs were processed in the left frontal sub-gyral areas (Figure 6). These results were obtained by using subtraction methods taking out the activated regions of interest (ROIs) in CA-matched controls from the ones in people with WS.

Results within-group comparisons revealed that CA individuals processed distinctly all types of semantically related word pairs except for the association and causal word pairs. This finding was supported for both 
types of words activating the left frontal gyrus. The only comparison reached significance in people with WS was between causal word pairs and functional word pairs, suggesting these two types of words were distinct in neural correlates. No difference yielded in other semantic related word pairs in the clinical group.
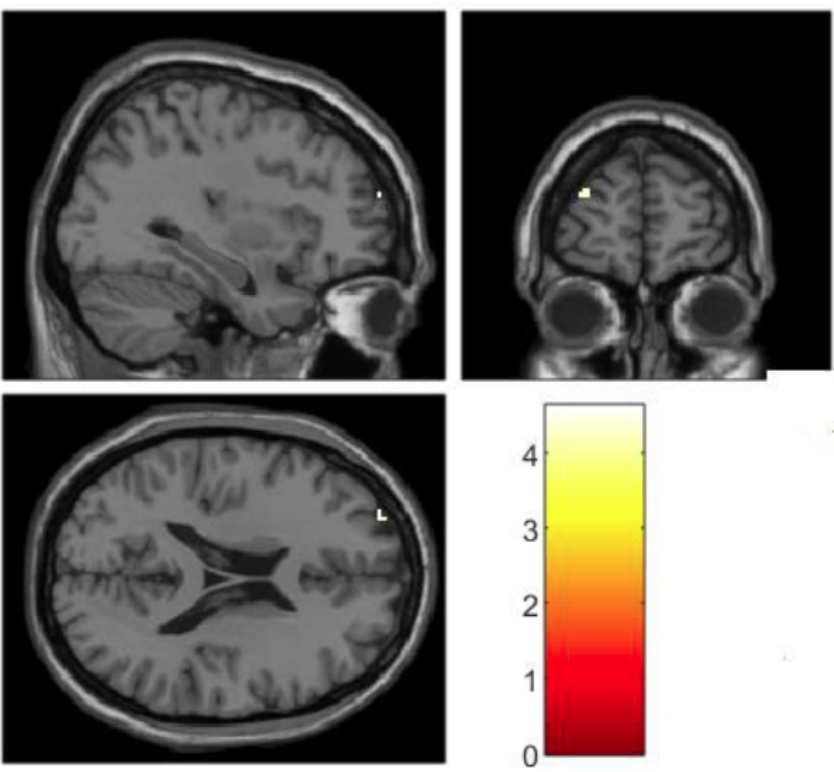

Figure 3: Subtracted brain area to causal word pairs: the left superior frontal gyrus.
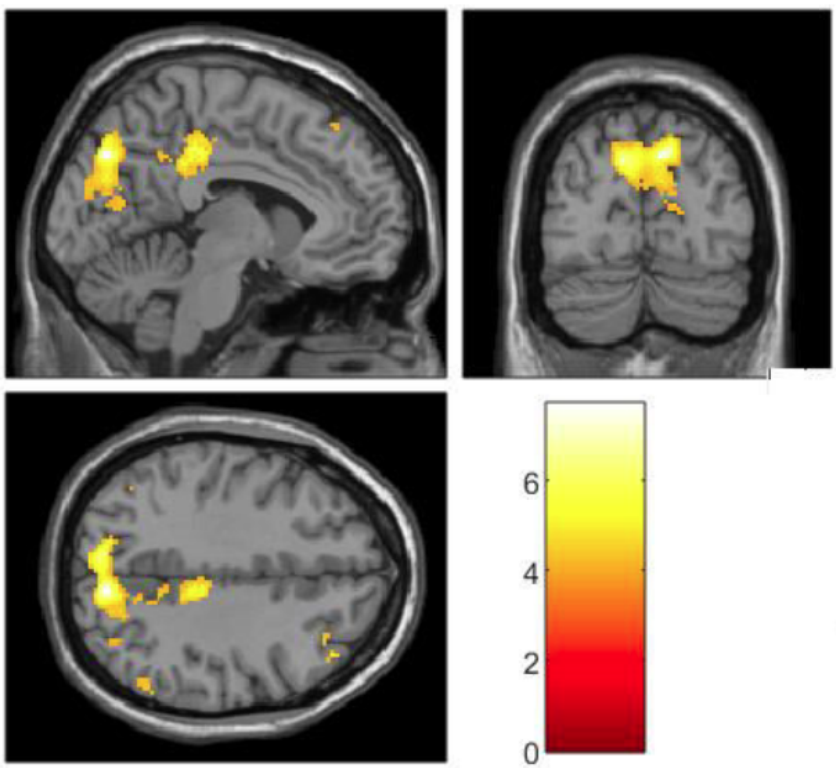

Figure 4: Subtracted brain area to categorical word pairs: the right parietal precuneus.

In an empirical review up to $275 \mathrm{fMRI}$ studies [33], the traditional brain areas related to language were the left prefrontal cortex (Broca's area) and the temporal cortex (Wernicke's area). However, different types of semantic relatedness would activate distinct brain
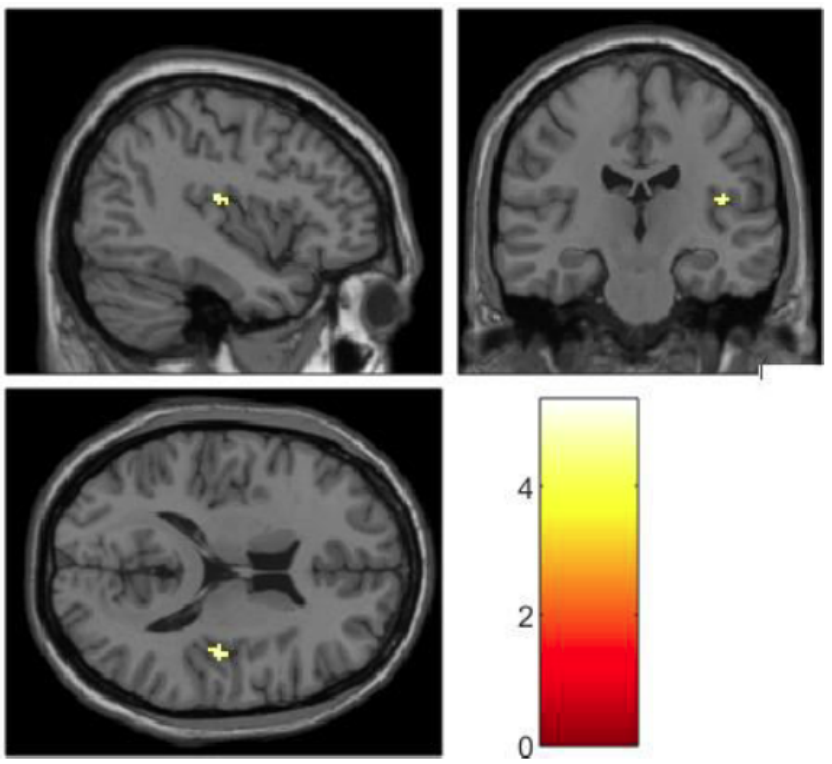

Figure 5: Subtracted brain area to functional word pairs: the right insula.
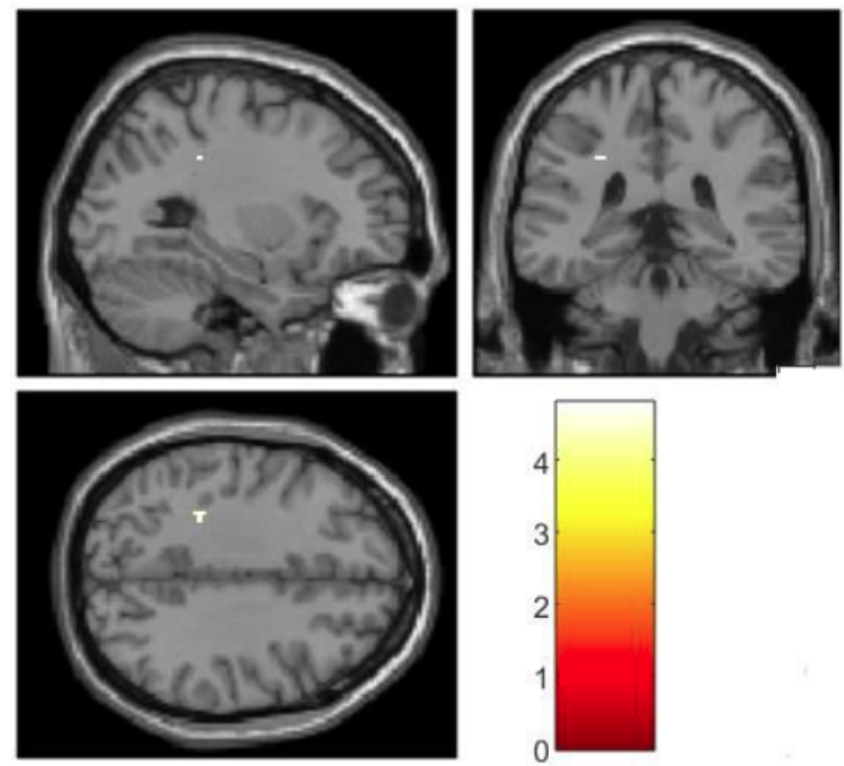

Figure 6: Subtracted brain area to associative word pairs: the left frontal sub-gyral areas.

areas. In the study by Satpute et al. [34], processing of English causal word pairs activated the dorsal lateral prefrontal cortex (DLPFC), whereas processing of English associative word pairs activated the superior temporal gyrus (STG). Based on the findings of previous studies, it was predicted that the left STG or Wernicke's area should be activated in examining comprehension of causal inferences and semantic priming in Chinese. However, the results for Chinese were different from those of English. For instance, the left superior frontal gyrus in processing Chinese causal word pairs and the left DLPFC was activated to English 
causal word pairs. The reason behind these differences may be the stimuli used in the two studies. In the study by Satpute et al. [34], the causal word pairs were weakly associative in meanings (e.g. moon vs. tide), but the associative word pairs were non-causal related (e.g. ring vs. emerald). Therefore, the causal word pairs were also associative word pairs, but it was not true vice versa. In the current study, subjective ratings were conducted on fine classifications of word types with distinct relatedness in the causal, categorical, associative, and functional linkage. This difference may result in distinct activations in the brain between the healthy controls.

\section{DISCUSSION}

This study aimed at examining neural correlates of causal inferences and semantic priming in people with WS to determine whether deviant semantic connections among words would emerge. If atypical processing patterns emerged, the deviant semantic connections among words might be the cause resulting in the difficulty of contextual integration in people with WS. A standard semantic priming task was conducted, and the differences of the healthy CA controls from WS individuals in responding to distinct word types were subtracted. Activation patterns in WS individuals differed from the ones in the healthy controls, indicating distinct processing to words linking in specific semantic relatedness. Semantic priming has its corresponding neural correlates. However, studies on semantic priming failed to define the relationship of probes and targets clearly. Rossell et al. [35] revealed that categorical word pairs (lemon vs. pear) but not functional word pairs (key vs. lock) involved less activation in the left anterior temporal cortex but stronger responses in the inferior parietal cortex. In the study by Sass et al. [36], words under the same superordinate (couch vs. bed, taxonomic words, sharing perceptual similarities) and words in various associative relationships (car vs. garage, thematic words, including causal, functional, and spatiotemporal relationships) were tested with a cross-modal method. The participants made lexical decisions toward their targets after hearing auditory primes. The behavioural results revealed that the priming effect was observed in processing thematic words but not taxonomic words. Moreover, unrelated taxonomic words involved less activation, but thematically related words activated larger responses in the left superior temporal sulcus. For taxonomically related words, the hippocampus, the middle cingulate gyrus, and the supplementary motor area were involved. The major responses involved in thematic processing were the left lateral temporal area, and the responses for taxonomic processing were in the right frontotemporal region. Sass et al. [36] demonstrated distinct neural correlates of thematic or taxonomic related semantic categories in tasks with auditory primes and visual targets. The current study contributes towards an advance understanding of semantic knowledge at the neurological level of people with WS by looking beyond behavioural studies and aiming to figure out the underlying cause of difficulty in contextual integration in people with WS.

To systematically investigate semantically related words, four types of word pairs in semantic relatedness were involved in this neuroimaging study: causal, categorical, functional, and associative. People with WS performed similarly to the healthy controls behaviourally with longer response latencies and lower accuracy in responding to casual word pairs than other word pairs. Neurological findings showed distinct processing areas for each type of words, suggesting different neurological validity of word types in semantic relatedness. Further comparisons between typically developing controls and individuals with WS revealed distinct brain processing areas. This pattern of normal behaviours but atypical neurological responses provides supporting evidence to the asymmetry of brain and behaviour. It indicates a compensatory strategy in the development of people with genetic deficits (semantic conceptual study in people with WS: Hsu et al. [20]; face processing in people with WS: Hsu and Chen [15]; face processing in Prader-Willis syndrome: Halit et al. [37]) and confirmation of neuroconstructivism that early mutation causes later atypical developments in language and cognition [38-42].

Neural correlates of semantic priming were also influenced by semantic relatedness and connectivity in semantic networks [43]. Wible et al. [43] showed that semantically related word pairs showed a reduction in the left frontal area and superior/anterior/middle temporal areas but were greater in the precuneus region compared to unrelated word pairs, which increased activation in the latter region. Moreover, bilateral lateral temporal areas were modulated by the level of connectivity or priming extent of word pairs.

An fMRI study examined the cohesiveness of narrative texts in discourse comprehension through reasoning reported different extent of causal relatedness triggered distinct activation strength [44]. Participants were asked to read two-sentence passages which were causally related in high, 
moderate, and low bonds. The ROls focused on the inferior, middle, and superior temporal cortex (Wernicke's area), inferior frontal cortex (Broca's area), and DLPFC. The results revealed stronger activation for moderately related passages in the right hemisphere compared to high and low related ones, suggesting more energies were involved in moderate causal related texts. A non-significant linear decrease in activation to relatedness in DLPFC was observed. Together, it was concluded that successful text comprehension requires bilateral DLPFC for the generation of inferences and the right hemisphere for the integration of inferences.

Causal inferences are integrated in the right hemisphere, including physical causal reasoning and linguistic causal reasoning. Physical causal reasoning requires perception and integration; hence, it is influenced by spatial and temporal contiguity. Incontiguity caused by spatial and temporal factors makes causal link less possible. Roser and colleagues [45] presented clips with the collision of two balls to two split-brain patients who were asked to judge whether collisions were causally related. Presentations through a single visual field were employed to investigate the hemispheric effect in causal inference. The results revealed that in the left visual field $(\mathrm{RH})$ display, the contiguity effect was larger than the display in the right visual field (LH). The percentages of non-causal judgments were increased to the larger spatial gap and longer temporal delay. This finding was consistent with Mason and Just [44] claiming causal inferences are integrated in the $\mathrm{RH}$. Roser et al. [45] contributed to confirm processing steps of physical causal inferences in perception and integration. Straube and Chatterjee [46] further conducted neuroimaging studies to find out stepwise neural correlates of causal inferences in space and time. Seven types of spatial contiguity and temporal contiguity each were taken as independent variables. Behavioural results were consistent with previous findings that causal judgments were made with smaller spatial gap and shorter temporal lag than non-causal ones. Neuroimaging results revealed no interaction of space and time, indicating independence of these two factors in causal inferences. While spatial angles were manipulated, the right postcentral area and the superior/inferior parietal cortex were activated; and while temporal delays were controlled, the left putamen (basal ganglia) was involved. These findings showed that causality inference is elemental in human cognition.

When comparing with another causality related to social contexts by subtracting physical causality in directional movements [47], neural correlates in common emerged, including the inferior and middle frontal areas, inferior parietal lobe, bilateral insula, and supplementary motor area. The only difference of social causality from physical causality was in the activation of the temporal parietal junction. Though the limitation of Wende et al.'s study was that the social contexts were artificial as the balls were personalised (Mrs. Red, Mr. Blue), which was far from a natural setting to probe real social causality.

In reading comprehension, verbs which imply inferences and coherence in texts are influential factors in generating causality. Virtue et al. [48] identified implicit texts but not explicit texts processed in the superior temporal gyrus (STG) and inferior frontal gyrus (IFG). More specifically, the right STG was involved in verbs key to inferences, and the left STG was essential to contextual coherence. Furthermore, participants who had larger working memory span activated posterior cingulate area while processing implicit texts than explicit texts, and the left STG with the left IFG involved at processing inferenced verbs and contextual coherence related information. The right STG was activated in processing inferenced verbs but anterior insula in coherence.

It is possible to conduct fMRI studies on people with intellectual disabilities. The results are interpretable because no correlations of intellectual abilities with low cognitive loading tasks were observed in fMRI studies [49]. In order to maximise the experimental effect on causal inferences and semantic priming in people with WS at the neurological level, some compromises were adjusted. Due to attention span limit of people with WS, some of WS participants received associative pairs and causal pairs prior to categorical pairs and functional pairs before they became too tired to pay attention. Healthy controls and other WS participants received fixed-order blocks in associative, categorical, causal, and functional word pairs. This subtle difference in presenting experimental blocks in the current study may have influenced the results, but still, the data obtained is valuable in the research field of neurodevelopmental disorders. It is truly not easy to conduct a neuroimaging study on people with WS who quite often are accompanied by physical difficulties and equipped with metal braces on their teeth or body armour to enhance their spinal strength in walking. Due to metal attachment in their bodies, it is impossible for WS individuals to receive scanning tasks. Hence, only a few participants with WS qualified to receive the test at the time in a small island like Taiwan, where the 
current study was conducted. Despite this subtle compromise, the study makes a good contribution to image neural correlates of causal inferences and semantic priming in people with WS and provides supporting evidence to the brain and behavioural asymmetry. It is confirmed that atypical neural network in processing lexical semantics is one of the causes resulting in impairment of contextual integration in people with WS. With neuroimaging technique examining the neurological processing of people with WS, this study highlights the importance of looking beyond behavioural studies in neurodevelopmental disorders [39].

\section{ACKNOWLEDGEMENTS}

This study was sponsored by the Ministry of Science and Technology, Taiwan (MOST 106-2410-H211-001) and the Fundamental Research Funds for the Central Universities, China, with start-up funding from Hunan University. The author thanks all participants with Williams syndrome and their families for supporting the study. Special thanks to the Research Center for Mind, Brain, and Learning at National Chengchi University, Taiwan, for conducting the neuroimaging study.

\section{DISCLOSURE OF INTEREST}

The author declares no conflict of interest.

\section{DATA AVAILABILITY STATEMENT}

Data are available upon request.

\section{REFERENCES}

[1] Korenberg JR, Chen XN, Hirota H, Lai Z, Bellugi U, Burian D, Roe B, Matsuoka R. Genome structure and cognitive map of Williams syndrome. In Bellugi U, George M St (Eds.) Journey from cognition to brain to gene: Perspectives from Williams syndrome. Cambridge, MA: MIT Press 2000; pp. 147-178.

[2] Strømme P, Bjømstad PG, Ramstad K. Prevalence estimation of Williams syndrome. Journal of Child Neurology 2002; 17(4): 269-271. https://doi.org/10.1177/088307380201700406

[3] Bellugi U, Lichtenberger L, Jones W, Lai Z, George M. The neurocognitive profile of Williams syndrome: A complex pattern of strengths and weaknesses. Journal of Cognitive Science 2000; 12(1): 7-29. https://doi.org/10.1162/089892900561959

[4] Semel E, Rosner SR. Understanding Williams syndrome: Behavioral patterns and interventions. Mahwah: NJ Lawrence Erlbaum and Associates 2003. https://doi.org/10.4324/9781410607416

[5] Tyler LK, Karmiloff-Smith A, Voice JK, Stevens T, Grant J, Udwin $\mathrm{O}$, et al. Do individuals with Williams syndrome have bizarre semantics? Evidence for lexical organisation using an on-line task. Cortex 1997; 33(3): 515-527. https://doi.org/10.1016/S0010-9452(08)70233-8
Wang P, Bellugi U. Williams syndrome, Down syndrome, and cognitive neuroscience. American Journal of Diseases of Children 1993; 147: 1246-1251. https://doi.org/10.1001/archpedi.1993.02160350120019

[7] Firth U. Autism and "Theory of Mind". In Gillberg C (Ed.) Diagnosis and Treatment of Autism. New York: Plenum Press1989; pp. 33-52.

https://doi.org/10.1007/978-1-4899-0882-7 4

[8] Hsu CF, Karmiloff-Smith A, Tzeng O, Chin RT, Wang HC. Semantic Knowledge in Williams Syndrome: Insights from Comparing Behavioural and Brain Processes in False Memory Tasks. Proceedings of the 6th IEEE International Conference on Development and Learning 2007; 6: 48-52. https://doi.org/10.1109/DEVLRN.2007.4354074

[9] Hsu CF, Tzeng JL. Contextual effect in people with Williams syndrome. Research in Developmental Disability 2011; 32(2): 781-787. https://doi.org/10.1016/j.ridd.2010.11.001

[10] Hsu CF. Contextual integration of causal coherence in people with Williams syndrome. Research in Developmental Disability 2013a; 34(10): 3332-3342.

https://doi.org/10.1016/j.ridd.2013.06.031

[11] Hsu CF. Cross-modal contextual coherence of semantic integration in people with Williams syndrome. Research in Developmental Disability 2013b; 34(12): 4319-4327. https://doi.org/10.1016/j.ridd.2013.09.002

[12] Hsu CF. Is the contextual effect weak in people with Williams Syndrome? An investigation of information integration ability using pictures. Research in Developmental Disability 2014c; 34(3): 932-939.

https://doi.org/10.1016/j.ridd.2012.11.015

[13] Hsu CF. Modality Effect of Contextual Integration in People with Williams Syndrome. Research in Developmental Disability 2014a; 35(7): 1571-1578. https://doi.org/10.1016/j.ridd.2014.03.049

[14] Hsu CF. Cross-domain investigation of weak centra coherence in people with Williams syndrome: Asymmetrical brain and behavioral performances in verbal and nonverbal domains. In Porter $\mathrm{OH}$ (Ed.) Semantic Memory: Neurobiology, Disorders and Therapeutic Strategies for Improvement. Nova Science Publishers 2014b; pp. 71-82.

[15] Hsu CF, Chen JY. Deviant neural correlates of configural detection in facial processing of people with Williams syndrome. Bulletin of Special Education 2011; 39(1): 61-84.

[16] Frith U, Happé F. Autism: beyond "theory of mind". Cognition 1994; 50: 115-132. https://doi.org/10.1016/0010-0277(94)90024-8

[17] Frith U, Happé F. Theory of mind and self consciousness: What is it like to be autistic? Mind and Language 1999; 14: 122.

https://doi.org/10.1111/1468-0017.00100

[18] Happé F. Autism: cognitive deficits or cognitive style. Trends in Cognitive Science 1999; 3(6): 216-222. https://doi.org/10.1016/S1364-6613(99)01318-2

[19] Happé F, Frith U. The neuropsychology of autism. Brain 1996; 19: 1377-1400. https://doi.org/10.1093/brain/119.4.1377

[20] Hsu CF, Karmiloff-Smith A, Tzeng O, Chin RT, Wang HC. Semantic Knowledge in Williams Syndrome: Insights from Comparing Behavioural and Brain Processes in False Memory Tasks. Proceedings of the 6th IEEE International Conference on Development and Learning 2007; 6: 48-52. https://doi.org/10.1109/DEVLRN.2007.4354074

[21] Roediger HL, McDermott KB. Creating false memories: Remembering words not presented in lists. Journal of Experimental Psychology: Learning, Memory, and Cognition 1995; 21: 803-814.

https://doi.org/10.1037/0278-7393.21.4.803 
[22] Pinheiro AP, Galdo-Alvarez S, Sampaio A, Niznikiewicz M, Goncalves OF. Electrophysiological correlates of semantic processing in Williams syndrome. Research in Developmental Disability 2010; 31: 1412-1425.

https://doi.org/10.1016/j.ridd.2010.06.017

[23] Hsu CF. Semantic Priming and Associative Priming in People with Williams Syndrome. In Duncan LT (Ed.) Advances in Health and Disease. Nova Science Publishers 2017; Vol. 2: pp. 171-196.

[24] Bransford J, Franks J. The abstraction of linguistic ideas: A review. Cognition 1972; 1: 213-248. https://doi.org/10.1016/0010-0277(72)90020-0

[25] Bransford J, Franks J. A brief note on linguistic integration. Journal of Verbal Learning and Verbal Behavior 1974a; 13(2): 217-219. https://doi.org/10.1016/S0022-5371(74)80046-0

[26] Bransford J, Franks J. Memory for syntactic form as a function of semantic context. Journal of Experimental Psychology 1974b; 103(5): 1037-1039.

https://doi.org/10.1037/h0037395

[27] Mills DL, Alvarez TD, George MS, Appelbaum LG, Bellugi U, Neville $H$. Electrophysiological studies of face processing in Williams syndrome. Journal of Cognitive Neuroscience 2000; 12(1): 47-64.

https://doi.org/10.1162/089892900561977

[28] Neville H, Mills D, Bellugi U. Effects of altered auditory sensitivity and age of language acquisition on the development of language-relevant neural system: Preliminary studies of Williams syndrome. In Broman S, Grafman J (Eds.) Atypical cognitive deficits in developmental disorders: Implications for brain function. New Jersey: Lawrence Erlbaum Association 1994; pp. 67-83.

[29] Hsu CF. Contextual Effects on Semantic Grouping in People with Down Syndrome. International Journal of Developmental Disability 2019; 65(2): 65-72.

https://doi.org/10.1080/20473869.2017.1353659

[30] Hsu CF. Contextual integration of causal coherence in people with Down syndrome: Evidence from figurative comprehension. Journal of Intellectual Disabilities-Diagnosis and Treatment 2016b; 4(1): 55-62.

https://doi.org/10.6000/2292-2598.2016.04.01.7

[31] Chen $\mathrm{RH}$, Chen $\mathrm{CY}$. Wechsler Scale Intelligence Test for Children. $4^{\text {th }}$ Ed. (Chinese Version). Taipei: Bookstore for Chinese Behavioral Science 2010.

[32] Chen RH, Chen CY. Wechsler Adult Intelligence Scale. $3^{\text {rd }}$ Ed. (Chinese Version). Taipei: Bookstore for Chinese Behavioral Science 2002.

[33] Cabeza R, Nyberg L. Imaging cognition II: An empirical review of 275 PET and fMRI studies. Journal of Cognitive Neuroscience 2000; 12(1): 1-47.

https://doi.org/10.1162/08989290051137585

[34] Satpute AB, Fenker DB, Waldmann MR, Tabibnia G, Holyoak $\mathrm{KJ}$, Lieberman MD An fMRI study of causal judgments. European Journal of Neuroscience 2005; 22: 1233-1238. https://doi.org/10.1111/j.1460-9568.2005.04292.x

[35] Rossell SL, Price C, Nobre AC. The anatomy and time course of semantic priming investigated by fMRI and ERPs. Neuropsychologia 2003; 41: 550-564. https://doi.org/10.1016/S0028-3932(02)00181-1
[36] Sass K, Sachs O, Krach S, Kircher T. Taxonomic and thematic categories: Neural correlates of categorisation in an auditory-to-visual priming task using fMRI. Brain Research 2009; 1270: 78-87.

https://doi.org/10.1016/j.brainres.2009.03.013

[37] Halit H, Grice S, Bolton P, Johnson MH. Face and gaze processing in Prader-Willi syndrome. Journal of Neuropsychology 2008; 2(1): 65-77. https://doi.org/10.1348/174866407X243305

[38] Karmiloff-Smith A. Crucial differences between developmental cognitive neuroscience and adult neuropsychology. Developmental Neuropsychology 1997; 13(4): 513- 524.

https://doi.org/10.1080/87565649709540693

[39] Karmiloff-Smith A. Development itself is the key to understanding developmental disorders. Trends in Cognitive Science 1998; 2(10): 389-398. https://doi.org/10.1016/S1364-6613(98)01230-3

[40] Karmiloff-Smith A. Atypical epigenesis. Developmental Science 2007; 10(1): 84-88 https://doi.org/10.1111/j.1467-7687.2007.00568.x

[41] Karmiloff-Smith A. Nativism versus neuroconstructivism: Rethinking the study of developmental disorders. Developmental Psychology 2009; 45(1): 56-63. https://doi.org/10.1037/a0014506

[42] Mareschal D, Johnson MH, Sirois S, Spratling M, Thomas MSC, Westermann G. Neuroconstructivism. Oxford University Press 2007.

[43] Wible CG, Han SD, Spencer MH, Kubicki M, Niznikiewicz $\mathrm{MH}$, Jolesz FA, McCarley RW, Nestor P. Connectivity among semantic associates: An fMRI study of semantic priming. Brain and Language 2006; 97: 294-305. https://doi.org/10.1016/j.bandl.2005.11.006

[44] Mason R, Just MA. How the brain processes causal inferences in text: A theoretical account of generation and integration component processes utilising both cerebral hemisphere. Psychological Science 2004; 15(1): 1-7. https://doi.org/10.1111/j.0963-7214.2004.01501001.x

[45] Roser ME, Fugelsang JA, Dunbar KN, Corballis PM. Dissociating processes supporting causal perception and causal inference. Neuropsychology 2005; 19(5): 591-602. https://doi.org/10.1037/0894-4105.19.5.591

[46] Straube B, Chatterjee A. Space and time in perceptual causality. Frontiers in Human Neuroscience 2010; 4: 1-10. https://doi.org/10.3389/fnhum.2010.00028

[47] Wende KC, Nagel A, Blos J, Stratmann M, Chatterjee A, Kircher T, Straube B. Differences and commonalities in the judgment of causality in physical and social contexts: An fMRI study. Neuropsychologia 2013; 51: 2572-2580. https://doi.org/10.1016/j.neuropsychologia.2013.07.027

[48] Virtue S, Haberman J, Clancy Z, Parrish T, Beeman MJ. Neural activity of inferences during story comprehension. Brain Research 2006; 1084(1): 104-114.

https://doi.org/10.1016/j.brainres.2006.02.053

[49] Pryweller JR, Avery SN, Blackford JU, Dykens EM, ThorntonWells TA. The effect of intellectual ability on functional activation in a neurodevelopmental disorder: preliminary evidence from multiple fMRI studies in Williams syndrome. Journal of Neurodevelopmental Disorders 2012; 4: 1-24. https://doi.org/10.1186/1866-1955-4-24

\section{DOI: https://doi.org/10.6000/2292-2598.2020.08.04.13}

(C) 2020 Ching-Fen Hsu; Licensee Lifescience Global.

This is an open access article licensed under the terms of the Creative Commons Attribution Non-Commercial License (http://creativecommons.org/licenses/by-nc/3.0/) which permits unrestricted, non-commercial use, distribution and reproduction in any medium, provided the work is properly cited. 